\title{
A Brief Review on EEG Signal Pre-processing Techniques for Real-Time Brain-Computer Interface Applications
}

\author{
Venkata Phanikrishna B ${ }^{1 *}$, Allam Jaya Prakash ${ }^{2}$, Paweł Pławiak ${ }^{3 \& 4}$ \\ ${ }^{1}$ Assistant Professor, VIT Vellore, Vellore, Thamilanadu-632014, India; \\ b.phanikrishna@gmail.com, ${ }^{2}$ Research Scholar, National Institute of Technology, Rourkela, \\ Odisha-769008 India; allamjayaprakash@gmail.com, ${ }^{3}$ Department of Computer Science, Faculty of \\ Computer Science and Telecommunications, Cracow University of Technology, Krakow, Poland; \\ pawel.plawiak@pk.edu.pl, ${ }^{4}$ Institute of Theoretical and Applied Informatics, Polish Academy of Sciences, \\ Gliwice, Poland; plawiak@iitis.pl
}

\begin{abstract}
Electro Encephalo Gram (EEG) is a monitoring method used in biomedical and computer science to understand brain activity. The neurological abilities, disabilities, and thinking of a person depend upon the signals generated by the brain. Therefore, the analysis and classification of these signals play a prominent role in estimating a person's behavior to certain events. Manually analyzing these signals is very tedious and time-consuming, so an automated scientific tool is required to analyze the brain signals. In this work, the authors are explored various pre-processing segmentation techniques that are helpful in an automatic machine and deep learning-based classification methods available for EEG signal processing. Most of the machine learning techniques are followed (i) pre-processing, (ii) feature extraction, and (iii) classification for analyzing the EEG signals. In contrast, deep learning techniques are followed only by pre-processing and classification steps. Manual feature extraction is not required in deep learning as machine learning methods, but pre-processing is common in both techniques. Extraction of the basic sub-band components from EEG signals such as delta $(\delta)$, theta $(\theta)$, alpha $(\alpha)$, beta $(\beta)$, and gamma $(\gamma)$ are very important in pre-processing stage. These sub-bands of EEG signal have extraordinary evidence related to multiple neurophysiological processes, which are useful for further prediction \& diagnosis of diseases and other emotion-based applications. This review paper elaborates various elementary ideas of extracting EEG sub-bands and the role of EEG in Brain-Computer Interface $(\mathrm{BCl})$ in classification.
\end{abstract}

Index Terms-Brain-Computer Interface $(\mathrm{BCl})$, classification, Electro Encephalo Gram (EEG), Pre-processing, Sub-band.

\section{INTRODUCTION}

$\mathrm{E}$ EG means ('E-ELECTRO') registration of Brain electrical actions, ('E-ENCEPHALO') getting the signal from Brain and ('G-GRAM') indicating in the form of a graph for representing the bio-electrical neural activity of the brain of any living being like human or animal [1], [2]. It contains valuable data associated with different physiological states of the brain, for examining the multiple health center applications like epilepsy, drowsy (sleepy) complaint, coma, brain injury, strokes, tumors, changes in behavior \& manages several disorders [1], [3], [4]. In early days, physicians used electrodes, amplifiers (to maximize the signal), mesh and machine hand (pen) to plot the EEG signal graph on a paper, as shown in Fig.1

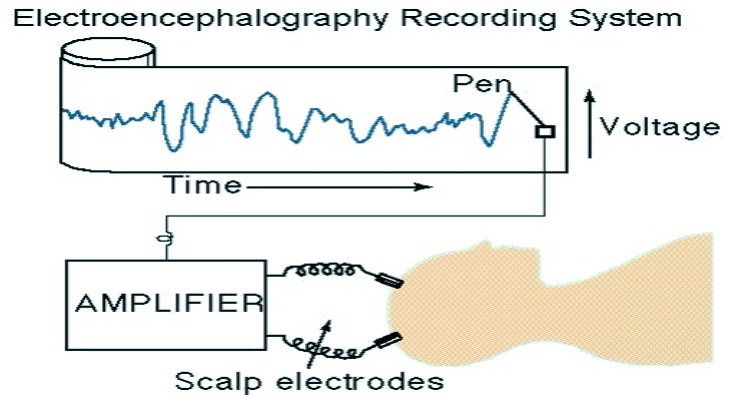

Fig. 1: Olden days EEG recording System.

The bio-electrical signals created by the cerebrum portray the status of the mind as well as the whole body. The advanced digital signal analyzing methods are recently used to analyze the EEG signal with the help of computers for digitization and storing for future analysis [5], [6]. EEG electrodes are disposable and recyclable which are made of silver or gold and may also use some other reusable materials like stainless steel or tin [7]. Some of EEG electrodes are Standard wet electrodes, Rigid metal electrodes, dry foam electrodes, MEMS sensors, micro-probe electrodes, non-contact sensors, epidermal electrodes etc. [7], [8]. Frequently used scalp electrodes contain an $\mathrm{Ag}-\mathrm{AgCl}$ disks, with the diameter less than $3 \mathrm{~mm}$ and it is flexible to plug into an amplifier. Some sample electrodes used in EEG cap is shown in Fig.2.

For academic research purpose, usually, wearable headbands or electrode caps are used as in Table 1 [7], [9]. 

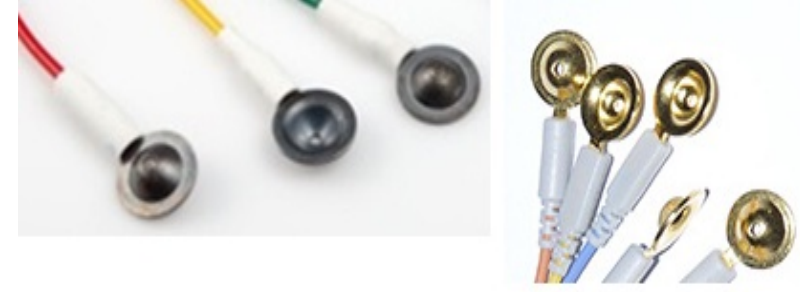

Fig. 2: Electrodes which made with Silver chloride or $\mathrm{AgCl}$ plated and with gold for EEG cap.

TABLE 1: Wearable Headbands for EEG signal Acquisition.

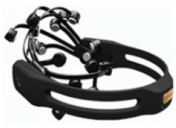

(a) Emotiv.

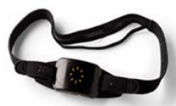

(c) Zeo

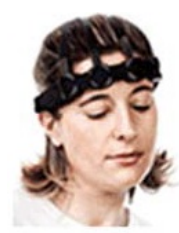

(b) StarLab.

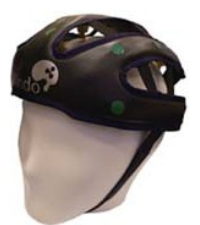

(d) Mindo 16.
Needle electrodes are those that must be embedded beneath the skull with insignificant invasive actions. If you get an EEG signal without any internal or external provocation, it is a natural EEG signal; otherwise, it is called Event-Related Potential (ERP) EEG signal. ERP-EEG signal used to analyze the time-based activity such as cognitive or motors are processed to study about physiological corresponds of mental procedure [10]. This type of signal has a different application, such as communicating individually without any motor system, course movement, epileptic seizures, Alzheimer's disease, etc. [11]. Independent Component Analysis (ICA) is one of the ERP-EEG signal analysis techniques for extracting the required ERP information and performing the classification. Auto-task Independent Components ERP method is the extension of ICA for extracting the independent components (IC) and classification. In this method, classification is done by gathering each IC's spectral, spatial, and temporal features [12], [13]. Analyzing the ERP-EEG signal, acquired under some positions like head body movements of humans, we can predict emotional feelings such as fear, happy or normal by using Linear Discriminant Analysis (LDA) [14].

\subsection{EEG signal challenges and its role in $\mathrm{BCl}$}

EEG signals regulate the body and specify the thinking and other abilities of the human being. By analyzing these signals we predict, what a person is thinking or wishing to do. Acquiring those signals without noise is a very difficult task. First applying conductive gel on electrodes and record towards the beginning of each session [15]. The major challenge of EEG is its dynamic nature, i.e., frequency com- ponents that are usually measured in terms of means and variances frequently change concerning time. So it is a challenging task to analyze the signal in both time and frequency domains. For analyzing such signals, some transformations such as Gabor transform ( Short-time Fourier Transform), winger, Choi-Williams distribution, wavelet transformation (WT), and multi-resolution time-frequency analysis (is a combination of WT and STFT) [16], [17]. Another method is decomposing the non-stationary signal into small piecewise stationary [18]. This signal is changed from time to time, and gathering these signals from the brain also plays a major role, especially in the case of transformation rate. Presently available systems transforming the EEG data from brain up to 10 to 25 bits per minutes [19], [20].

The signals that are acquired from the brain are small in size (microvolts). Analysis of such a tiny signal is very difficult and may not get the required result perfectly. These small amplitude signals are more likely to be detected by background noise like human body movement, cable connection problems, or other power fluctuations. To detecting \& reducing such noises and analyzing according to our application requirement, amplification is required to maximize the strength of EEG signal [21]. Brain-computer Interface (BCI) is a branch of Human-Computer Interfaces $(\mathrm{HCI})$, provides interaction between the human brain and computer using non-muscular channels. This communication system scrutinized the brain signals and translated them into commands for output devices. The classification of the tasks can be implemented with the help of a machine and deep learning methodologies.

$\mathrm{BCI}$ provides strength to disabled people by reducing dependency factors of their daily work. This can be done by controlling the surrounding environment without anyone's help, for instance, cognitive tasks like driving wheelchairs and logical controls (volume control), semantic mapping, etc. [22],[23]. This system detects the specific patterns in a person's brain activity to recognize what that person intended to do. EEG signal is one of the useful data in BCI for predicting brain activity like PET (Positron Emission Tomography), MEG (Magnetoencephalography), fMRI (Functional Magnetic Resonance Imaging)[24], [25]. As compared to other brain signals, EEG is best for BCI because it less expensive and less space required to gather the brain data [26],[27].

Based on the acquired data from the brain, the BCI system was categorized into two types: invasive and NonInvasive BCI's. In an invasive BCI system, gather the brain data from deeper regions of the brain. For this, electrodes are implanted under the skull of a human head surface. The data gathered from implanted electrodes is called "electrocorticography" (ECoG). Positions of implanted electrodes are based on the application of the BCI system. For considering left vs. right-hand movement, one 64-grid right hemisphere, and two 4-strip inter-hemisphere are required. For only considering small left finger vs. tongue movement, 64-grid right hemisphere is sufficient, and for the small finger of right hand vs. tongue, one 20-grid central and four 16-strips frontal are required [28].

In a non-invasive BCI system, the gathering of brain data is done by placing electrodes on the surface of the head. In this scenario, BCI gathered brain data in EEG. Non-invasive 
$\mathrm{BCI}$ system preferred in monitoring conditions like cerebral palsy (brain injury), muscular dystrophy (muscle proteins are damaged), spina bifida (congenital condition, spine fails to close properly during the first month of pregnancy), amyotrophic lateral sclerosis (a disease that prevents neurons from sending impulses to the muscles), arthritis (people with arthritis are unable to use a keyboard and mouse), Parkinson's disease and Essential tremor (related to central nervous system disorder).

Some current research work shows that we can directly monitor the robots by using electrical activity of the brain gathered by microelectrodes. ASPICE project (Assistive System for Patient's Increase of Communication, ambient control and mobility in the absence of muscular Effort) related work to help the patient who suffers from spinal muscular atrophy is done with the help of non-invasive BCI system [29]. This type of communication system helps the patients suffering from motor disabilities do their work without any human intervention. BCI system is not limited to monitoring the robots. It also provides textual communication through spelling with steady-state visually evoked potentials (SSVEP). For this high information transfer rate (ITR) is required. This can be achieved with the help of EEGbased BCI; it has the capability of 5.32 bits per seconds ITRs and analyzes the frequency phase of 40 characters in half a second [30]. This type of communication helps the patients who are suffering from motor disabilities with other people.

The main difference between these BCI's is the signal resolution. In the case of invasive, we get the signal with less noise and better resolution than the non-invasive. In EEG-based BCI uses EEG signal from the brain scalp; this method is the reproducible and low-cost setup used to increase human capabilities beyond the normal level. The main advantage of EEG is non-invasive, less cost, and we recorded it easily from different perspectives like clinical, lab studies. But there is a drawback in EEG during the recording process. Artifacts (noise) in gathered EEG signal data lead to a high possibility of false alarm and unintentional control of the device. Artifacts can be categorized into two groups such as internal (physiological) and external artifacts. Internal artifacts related to the physiological activity of subject like ocular(related to the eye such as blinking, movement, flatter), cardiac(heart beats change), muscle movements (chewing, talking, sniffing and swallowing), sweating, hearing sudden sounds etc. External artifacts sources are environmental interfaces like hardware problems (electrodes displacement, cable movements, and damage), interference ( power fluctuation), and movement(head, body, limbs, and other movements of the subject) [31].

External artifacts are reduced with the help of the latest hardware technologies and interface methods like advanced wireless technologies and power management techniques. But internal artifacts are not possible to avoid using technology, and we must detect such artifacts and remove them externally. Among all internal artifacts, ocular artifacts create a serious problem because, without any movement of an eye, the subject is unable to stay for a long period[32]. To removing such artifacts from EEG signal, there are two algorithms such as Recursive Least Square (RLS) and Blind Source Separation (BSS), preferred by many researchers. BSS is best when we know which type of EOG is present [33]. Along with these two algorithms, there are some other methods for removing ocular artifacts from EEG, such as Independent component analysis (ICA), Discrete wavelet transform (DWT), Adaptive Noise Cancellation (ANC). Combination of multiple methods such as DWT and Adaptive predictor Filter (APF) provide better results compared to the other methodologies [34]

BioSig is a free software for analyzing the biosignals such as EEG, ECoG, ECG, EOG, and EMG. By using this software, we can remove artifacts from EEG. Spatial Filter, blind source separation regression are methods highly used for EOG reduction. In the case of a small number of EEG channels, principle component analysis (PCA) and Independent component analysis (ICA) performance is less than the regression analysis. EOG artifacts are in two categories: fast EOG artifacts (high amplitude) and slow artifacts (low amplitude). Regression-based EOG reduces both types of EOG artifacts irrespective of EEG channels [35]. By using blind source separation (BSS), we can reduce both muscle and eye movement artifacts[36].

This paper presents the literature review, such as positioning of electrodes, sub-bands of EEG, and different methodologies for generating sub-bands, which are explained in section-2. In section-3, we compare the results of different methodologies of sub-band generations with advantages and disadvantages. Lastly, we concluded in section-4.

\section{Literature Review \\ 2.1 Positioning of Electrodes}

To gather EEG signals from the brain placement of electrodes on the head scalp plays an important role. This can be done with the help of some reference points on the scalp, such as nasion (Nz), Inion(iz), Left preauricular point (LPA), and right preauricular point (RPA). The "10-20 System" is the first electrode placement system for gathering EEG signals from human scalp given by jasper in 1958 and is accepted by many researchers [37]. The number of electrodes required to gather the EEG signal is depended on the type of experiment and placing of the human subject like in the clinic or any other general place such as research labs etc. With the help of potential functions on the hemispherical surface and sampling theorem, we can specify the number of electrodes required [38]. The placement of the electrodes follows some topographic for getting tomography of brain area for covering all parts of the brain. The initial topographic system is 10-20 system which is designed by jasper in 1958 (shown in Fig. 3) and accepted by both ACNS (American Clinical Neurophysiology Society) previously known as AES (American Electroencephalographic Society)and IFCN (International Federation of Clinical Neurophysiology) previously known as IFS (International Federation of Society).

From this topographic system, researchers and scientists design other topographic systems such as the 10-10 system and the 10-5 system to cover the brain area completely. In the case of the "10-10 system," chattrian's 10-10 system contains 81 electrodes, IFCN 10-10 system contains 64 electrodes, ACNS 10-10 system contains 75 electrodes, and Oostenveld's 10-10 system contains 85 electrodes. IFSN and ACNS 10-10 systems are almost the same, and the only difference 


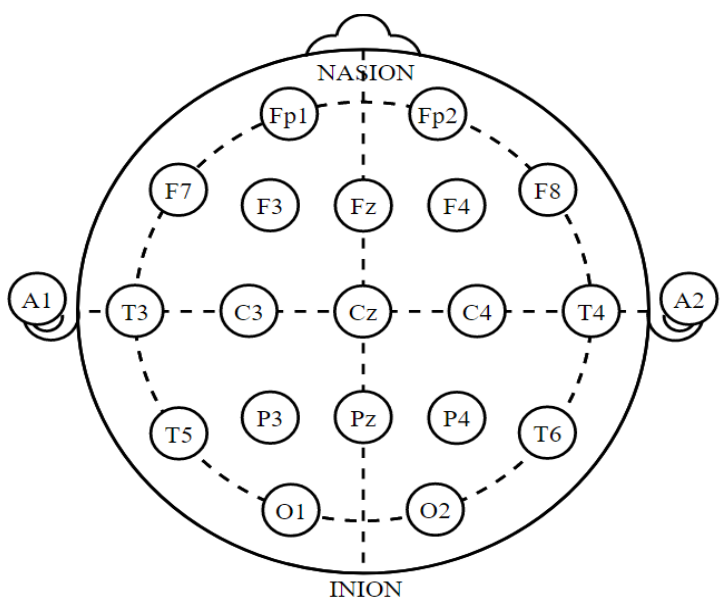

Fig. 3: Jasper's 21 Electrodes 10-20 system [13].

is IFCN follows jasper's topography for T3 to T6, and ACNS used T7, P7, T8, and P8. There is another 10-10 system called as unambiguously illustrated (UI) $10-10$ system [37]. Dr. Jasper built up a few rules for setting these electrodes; every anode position must determine the landmark, and it should be proportional to the size of the skull. These situations must cover all parts of the head. Electrodes appointed name not just to separate it from different electrodes and determines which part of the brain it covers[39]. The 10-10 system also called as "10\% system" (shown in Fig 4). The chatrian "10\% system" adds extra 60 electrodes to the 21 electrodes 10-20 system given by Jasper. The arrangement of the position of the new electrode follows Jasper rules only. Still, few electrodes are placed according to the boney structures other than the specified marks used in the 10-20 system to cover all relevant head regions [40].

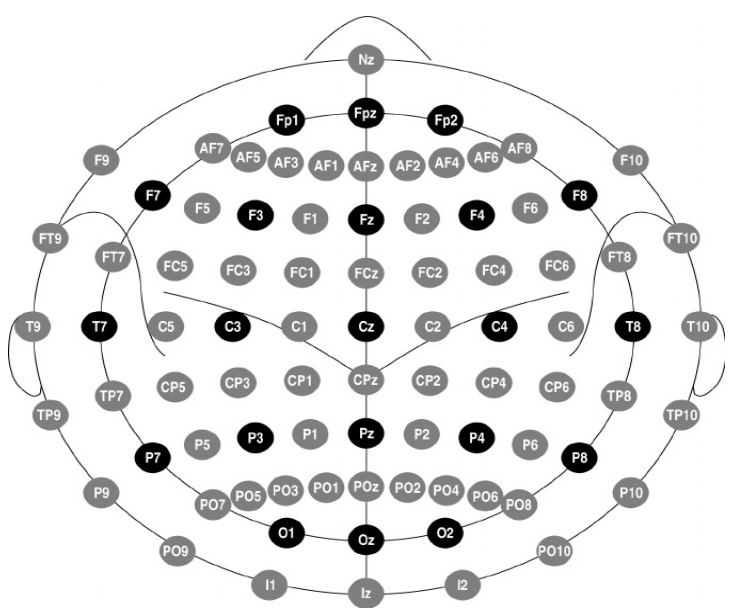

Fig. 4: $10-10$ or $10 \%$ system (Black circles related to $10-20$ system and extension to $10-10$ system is indicated with gray color circles) [8].

In some problem identification, it is compulsory to get high-resolution ERP-based EEG signals to analyze brain activity; in such cases, they may require more than 100 channels of EEG signal. To accomplish this requirement, there is a new extension of the 10-20 system called a 10-5 system or "5\%-system" (Shown in Fig. 5). 10-10 system is an extension of the 10-20 system, and the 10-5 system is the extension of the 10-10 system, and it uses a proportional distance of $5 \%$ of skull area to allow 345 electrodes locations. These many locations can be possible by using naming of the geographical direction of a compass-like a place between north and west in the direction of north-west[41].

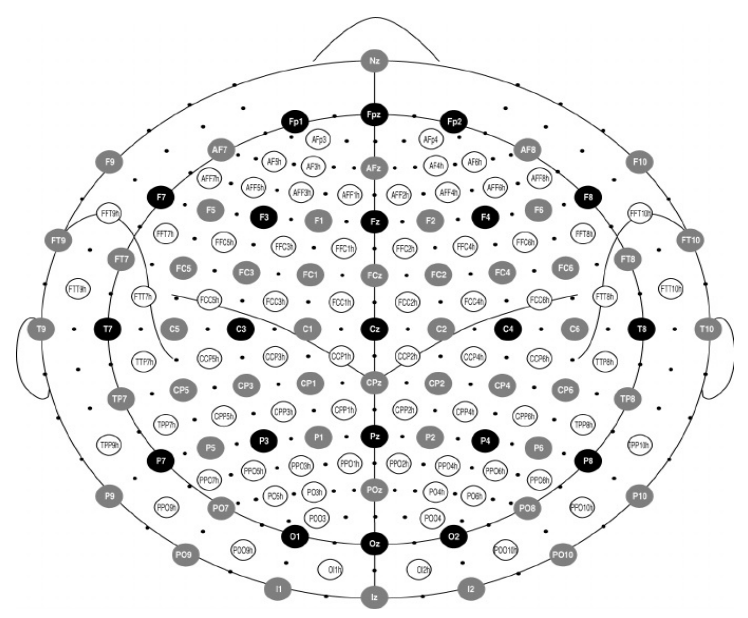

Fig. 5: $10-5$ or $5 \%$ system [8].

To acquire an EEG signal from the human brain, there is no need to use all locations provided by the 10-20 system, and the strength of EEG recording depends not only on the geographical coverage of the brain. Along with electrodes, the EEG signal collected from machines such as computers or any processor plays an important role. With the help of DSP processor DS-1104, they collect the EEG signal by using three electrodes; among them, one is placed at the forehead center, and another two are placed at the left and right of the forehead. Based on the application, we select the locations of 10-20 systems to gather the EEG signal. For instance, to analyze the right or left finger movements, we use c3 and c4, respectively; for ERP p300, we use F3, F4, P3, and P4 [42]. If any researchers design a new system is like 10-10,105 systems for acquiring EEG signal from the brain or any vendor sell any EEG acquired system which meets the IFCN standard, they must be approved by IFCN [43].

Considering the cost and arrangement of sensor nodes on the brain scalp, many hospitals use 21 electrodes "1020 System" to read EEG data from patients to analyze the brain working system. Fig. 4 shows one of the wired "1020 System" with 21 electrodes. For non-research people to place the electrodes on the brain, there is manual [44]. The electrodes used in "10-20 System" of Fig. 3 are differentiate by using some letters called F, T, P and O (shown in Table 2). These letter indications specify the different brain lobes as shown in Fig. 6, to show the different brain functions [45]. The electrode with the name " $\mathrm{C}$ " is only for identification purposes.

There is another electrode positioning system for EEG signals called "Maudsley." It is the modified version of "10-20 System", in which they use lowered enable better capturing of the signal, to capture the epileptic foci in epileptic seizures recordings. The main advantage of the 'Maudsley' system is a widespread analysis of the lower part of the cerebral convexity [46]. 


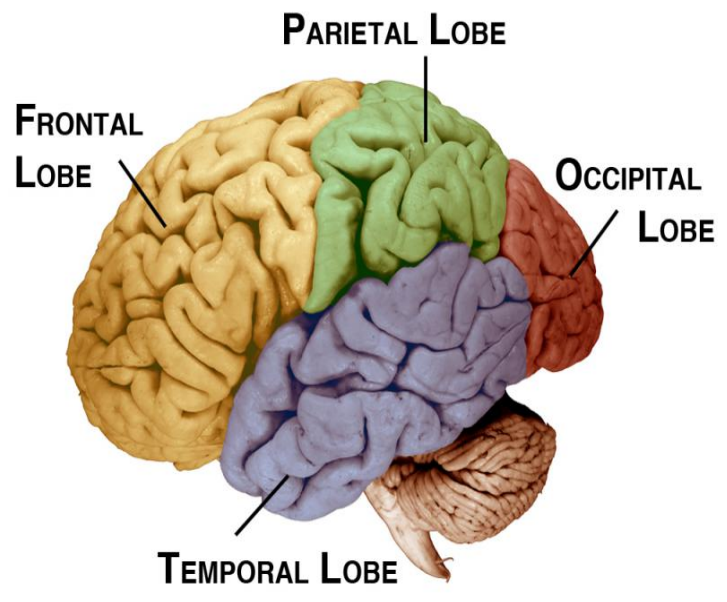

Fig. 6: Brain with lobes Identification [10].

TABLE 2: Brain lobes.

\begin{tabular}{|l|l|}
\hline Electrode & Lobe \\
\hline F & Frontal \\
\hline T & Temporal \\
\hline C & Central \\
\hline P & Parietal \\
\hline O & Occipital \\
\hline
\end{tabular}

\subsection{Sub-bands of EEG}

EEG signals regulate the body and specify thinking abilities. The bio-electrical signals created by the cerebrum represent the working of the mind. It contains valuable data to examine multiple clinic applications corresponding to epilepsy, drowsy complaints, head injury, strokes, tumors, changes in behavior, and other issues. For inspecting these things, we study the waves of EEG signals. Usually, these signals are non-stationary, so that they will change from time to time and person according to their states, such as alertness and sleep. The features of brain waves change with age, so, for predicting the human task based on the EEG signal, we investigate the brain waves in deepness. EEG signals have multiple sub-bands to examine the brain based on different frequency ranges [47]. These are Delta $(\delta)$, Theta $(\theta)$, Alpha $(\alpha)$, Beta $(\beta)$ and Gamma $(\gamma)$. Nature these sub-bands are specified in the Table 3 . The spectrogram has been utilized as a part of a wide variety of EEG applications because of its ability to clearly showing changes that happen in the rhythmic activities of the EEG, which generally would be hard to see from the time-space signal.

After generating these sub-bands from EEG, they use any of one sub-band or collection of two or more sub-bands based on researcher requirement. Utilizing alpha and theta cerebrum wave preparing might be a helpful intercession for the abstinent alcoholic [48]. By analyzing these sub-bands of EEG signals, we can predict the nature. In most of the cases, they use only delta, theta, alpha, and Beta. In rare cases, gamma signals are used; otherwise, it treated as noise signals. Each sub-band has its own property and useful for analyzing particular applications [49],[50]. Delta $(\delta)$ 0.5-4 Hz: The low-frequency brain waves were discovered by W. Grey Walter in 1936s and announced in 1963. During the reading of Brain waves for every 2 or 3 seconds, delta wave happens only once and mostly arises in a deep sleep. The features of these waves are the highest in amplitude and slowest waves used to predict the deep sleep and hyper-relaxed mind states. By analyzing these waves, we can predict the depth of anesthesia with the help of this wave power, and bispectral index [51]. These are mainly used to measure the sleep cycles, especially in stage 3 \& 4 [52]. Theta $(\theta) 4-8 \mathrm{~Hz}$ : It is the most significant wave in the whole place of brain activity. Frequently found over the brain's parietal and Temporal lobe region, announced by Wolter and Dovey in 1944. By analyzing these waves, we can predict the attention of a person [53].

These waves place a vital role in predicting women self-injurious behavior. Theta waves movement have been linked with person present states like imaginative thinks and deep meditation, light sleep, emotion, daydreaming, and susceptibility to hypnagogic states (placed in between wakeful and dropping asleep) and putting attention to solving particular task [54]. The theta waves gathered from the frontal mid-line area have specific activity-related things such as arithmetic addition and intelligence test. Such theta wave is call as $" \operatorname{Fm}(\theta)$ " [55]. The features of $\theta$ wave are gradually lower in frequency over long periods if the subject is in meditation or a snooze. These waves are energetic in the awakening adult. If the $\theta$ waves are irregular, it may be of various difficulties of a subject like tensions, etc. Theta state is mainly used to predict the Also used to recognize the memory and reductions in stress. If the theta wave increase in the same place, the delta and alpha waves also increase.

Alpha $(\alpha)$ 8-13 Hz: This wave is similar to the Delta wave, appears in all parts of the posterior half of the brain, and mainly occurs at the occipital region of the brain [56]. Introduced by Berger in 1929 [1]. Alpha waves normally look like a round or sinusoidal molded signal; we can specify both relaxed mindfulness without any consideration. This wave of the subject is produced in two ways, eyes open and close. Suppose eyes are closed, then scanning pattern produced by the visual region of the brain. If eyes are opened, and the signal is based on hearing unfamiliar sounds, by anxiety, or mental concentration or attention [57]. These waves also play a vital role in predicting memory performance. Based on frequency range alpha waves, there are two types: upper alpha $(10-13 \mathrm{~Hz})$ and lower alpha $(8-10 \mathrm{~Hz})$ bands. The upper alpha band is useful to analyze memory-related tasks, and for analyzing the attention process, the lower alpha band is more useful. Alpha frequency is high for good memory humans as compared to the bad memory human[58].

Beta $(\beta)$ 13-30 Hz: This wave has primarily occurred at frontal and central lobes of the brain, introduced by Berger in 1929 [1]. The amplitude of beta rhythms is under $30 \mathrm{mV}$. Beta wave with low magnitude frequency represents the busy mental state. By analyzing these waves, we can predict the status-quo (present state or condition) of humans; through this, we can help abnormal people. Beta wave analysis is used to control the motors [59]. These brain waves may be absent or reduced in brain-damaged areas. These waves changed situation to situation like gripping something and force parameters and also changed in case of preparing movement and executing such movements[60]. Gamma $(\gamma)>30 \mathrm{~Hz}$ : It Signifies the similarity of known ob- 
TABLE 3: EEG Sub-bands.

\begin{tabular}{|c|c|c|c|c|}
\hline Rhythms & $\begin{array}{l}\text { Frequency } \\
\text { Range }\end{array}$ & Location & Occurrence Reason /Useful area & Nature \\
\hline $\begin{array}{l}\text { Delta } \\
(\delta)\end{array}$ & 0.5 to $4 \mathrm{~Hz}$ & Frontal Lobe & $\begin{array}{l}\text { In deep sleep. Useful to measure the sleep } \\
\text { cycles }\end{array}$ & \multirow[t]{2}{*}{ Slow Waves, low frequency and high in amplitude } \\
\hline $\begin{array}{l}\text { Theta } \\
(\theta)\end{array}$ & 4 to $8 \mathrm{~Hz}$. & $\begin{array}{l}\text { Central fissure, } \\
\text { Temporal }\end{array}$ & $\begin{array}{l}\text { In meditation, light sleep. Useful to measure } \\
\text { the drowsiness of a human }\end{array}$ & \\
\hline $\begin{array}{l}\text { Alpha } \\
(\alpha)\end{array}$ & 8 to $12 \mathrm{~Hz}$. & Frontal, Occipital & $\begin{array}{l}\text { Used to measure mental concentration or } \\
\text { attention }\end{array}$ & \multirow[t]{2}{*}{ Fast Waves, High frequency and low in amplitude } \\
\hline $\operatorname{Beta}(\beta)$ & 12 to $30 \mathrm{~Hz}$. & Frontal, central & $\begin{array}{l}\text { For every thinking. Useful to measure brain } \\
\text { damaged area. }\end{array}$ & \\
\hline
\end{tabular}

jects, introduced by Jasper \& Andrews (1938) [1]. Recording of these waves was difficult. The advantages of analyzing the gamma waves are attention level calculation and cognitive phenomena. With the help of gamma power, we can predict the functional memory status and learn things. The irregularity in gamma waves indicates some neurological disorders like Alzheimer's and epilepsy [61]. The nature of this wave, such as high frequency and low amplitude, causes quick movement. This helps to enhance cognition. For those who have the highest IQ scores, their gamma waves are also high. [62].

\subsection{Methodologies for generating sub-bands}

To generating EEG sub-bands such as $\delta, \theta, \alpha, \beta$ and $\gamma$ there are many methodologies in which we specify some methodologies such as Fourier Transform, Digital Filters, and Wavelet Transform.

\subsubsection{The Fast Fourier Transform (FFT)}

It is one of the most popular digital signal processing (DSP) techniques for converting the one domain signal to another domain signal such as time to a frequency domain and vice versa, by using two methodologies called DFT (Discrete Fourier Transform) and Inverse DFT [63]. Sample conversion is presented in Fig.7.
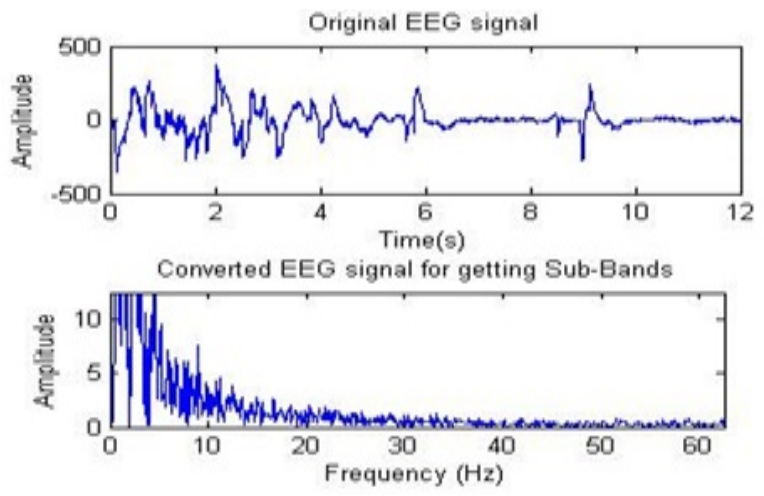

Fig. 7: Raw EEG data (first graph) with time domain and Frequency domain EEG data (second graph).

For performing this process, we have some predefined functions in the Matlab, such as "fft" and "ifft" [64]. This technique allows for the separation and studies of the different EEG frequencies for analyzing the EEG signal. FFT technique breaks down the signal into small components (follows the divide and conquer rule). To extracting the EEG sub-bands, the FFT decomposes the EEG signal into sins and cosines waves of different frequencies[65]. FFT is easy to use, and computing time is low because it reduces the number of computations from $N^{2}$ to $N \log _{2} N$. Here $\mathrm{N}$ is the size of a problem; for our EEG signal, we consider it as several samples[66]. FFT for continuous-time signal $x(t)$ is $X(f)$ is shown in below equation.

$$
X(f)=\int_{\infty}^{-\infty} x(t) e^{-j 2 \pi f t} d t
$$

The FFT is not only limited to extract the EEG sub-bands. It is also useful for extracting statistical features like power spectral density, spectral centroid, spectral energy, etc. [67]. The simple FFT is limited to the stationary signals because FFT only analyzes the continuous signal with uniform frequency. For non-stationary signals, FT provides Short-time Fourier Transform (STFT, also called Gabor Transform) with an approximated window to decompose the signal into piece-wise stationery. It is a time-frequency representation of the time-based EEG signal[68]. An indication of STFT is similar to the FFT. Here, the only difference is an extra windowing function $\omega(t)$ and whose positions are translated in time by $\tau$.

$$
\operatorname{STFT}(f, \tau)=\int_{\infty}^{-\infty} x(t) \omega(t-\tau) e^{-j 2 \pi f t} d t
$$

\subsubsection{Digital Filter}

Digital Filter is frequently used and executed in relationships with the recurrence relations that describe in what way the output signal is linked to the input signal, for example,

$y[n]=\frac{1}{a_{0}}\left(b_{0} x[n]+b_{1} x[n-1] \ldots b_{p} x[n-p]-a_{1} y[n-1]-a_{2} y[n-2]-\ldots-a_{Q} y[n-Q]\right)$

Here $\mathrm{P}$ and $b_{\mathrm{i}}$ are feed-forward filter order \& coefficients. $\mathrm{Q}$ and $a_{\mathrm{i}}$ is the feedback filter order \& coefficients, $\mathrm{x}[\mathrm{n}]$ and $y[n]$ are input and output signal respectively. Without Filters concept of digital signal processing, many fragments of EEG would be incomprehensible. The utilization of Filters can influence the EEG motion in ways that range from the unpretentious to the emotional. The principal advantage of filters is that they can clean the EEG to make it simpler to understand. The frequencies of all mind electrical action of intrigue exist in a specific data transfer capacity called bandwidth. EEG filters are set up with the goal that one filter rejects the larger part of the high-frequency action, and another filter rejects the lion's share of low-frequency movement. The scope of frequencies between these undesirable high and low frequencies permitted to go through the filter setup is alluded to as the band-pass. [5]. Digital filter has two models such as FIR and IIR for filtering EEG signal into various bands [69]. 


\subsubsection{Finite impulse response (FIR) Filter}

FIR filter is used to implement almost any type of digital frequency response. The FIR filter design can be done in two ways, such as frequency sampling and windowing FIR filters. In the case of frequency sampling method, filter design uses Inverse Discrete Fourier Transform (IDFT) for desired frequency response for filter coefficient. In the windowing method, FIR filters are determined by Inverse Discrete Time-Frequency Transform (IDTFT).FIR filter with frequency method is similar to the FFT. Both use the Discrete Fourier Transform, and representation is also easy. The problem of this design is the length of the filter is long because the IDFT of the sampled frequency response with the number of samples is equal to the number of filter coefficients if we using frequency sampling method [70]. The IDTFT would be a continuous frequency function, and it calculates the result of the impulse response (which is generated by IDFT) for the windowing filter. The IDTFT process is an estimation of the multiplication of impulse response by a rectangular window. Due to this reason, for extracting sub-bands from EEG signal prefer windowing based FIR filter, it is time-domain representation. Here for all the passband frequencies ideal frequency response of a filter is one, and for stopband frequency, the ideal frequency is zero. There are many windowing functions for a Windowbased FIR filter to extract the required frequencies from EEG signals. Those are the Rectangular window, Bartlett window, Hanning window, Hamming window, Blackman window, and Kaiser window [71]. In the case of EEG signal analysis among Blackman, Hamming, Hann, and Rectangular windows, Blackman window is better if we consider stopping band attenuation and transitivity bandwidth [72], if you consider all five windows, Kaiser window is best according to main and side lobes, and SNR [73]. The difficulty of FIR is EEG signal sampled at $1000 \mathrm{~Hz}$ to mine the Delta, Theta, alpha, and beta waves.

\subsubsection{Infinite Impulse Response (IIR) Filter}

These filters are recursive, means it uses current input and previous output samples for computing current output sample. IIR filters are considerably more effective than FIR filters [74]. There are many IIR filters: Butter-worth, Chebyshev (Type I \& II), and Elliptic IIR filters. To analyze EEG, commonly used IIR filters are Butter-worth, and elliptic IIR filters [75]. With the help of IIR, we can extract five main sub-bands of EEG signal with the help of relating to lowpass and band-pass IIR. This process is opposite to the Finite impulse response Filter. The generated sub-bands of EEG signals are shown in the next section.

\subsubsection{Wavelet Transform}

WT is a mostly used Digital signal processing method to perform time-frequency analysis of signals by providing a unified framework for different techniques for various applications such as telecommunication and biology. Because of their non-stationary signal analysis property, this method is a powerful method and alternative to the Fourier methods for analyzing EEG signal [76]. The appropriate possessions of wavelets have been utilized as a part of the investigation of medicinal signs such as EEG and ECG to identify the abnormalities and sudden changes in heartbeats [77]. WT can analyze the short-duration signal changes; due to this property, it is more popular for examining the electrophysiological signals of humans as compared to the other signal processing methods. Especially investigate the pulsed electromagnetic field (PEMF) in case of extremely low frequency (ELF) of ECG, EEG, and PPG (Photoplethysmography) analysis its plays major role. This analysis was done with the help of multi-resolution decomposition[78]. In wavelet transmission, a wavelet is an oscillation function whose energy is concentrated in time to represent transient, non-stationary signals better. WT working is done by two operations such as scaling and translation to observing the correlation between observed signal \& the basis function.[79]. As with FFT analysis, WT also expands the function in terms of basic function but not in terms of trigonometric polynomials (in FFT) but in terms of wavelets generated by mother wavelet for translate \& dilations [80]. Digital signal processing provides two types of wavelet transform such as Continuous Wavelet Transform (CWT) \& Discrete Wavelet Transform (DWT) [80], [81]. Depending on the application, one may be preferred to the others.

\subsubsection{Continuous Wavelet Transform (CWT)}

The input signal is continuous, and it has continuous parameters like time and scale, then CWT is best for extracting required features. CWT can be expressed as

$$
\begin{gathered}
C W T(a, b)=\int_{-i n f}^{i n f} x(t) \psi_{a, b}^{*}(t) d t \\
\psi_{a, b}(t)=\frac{1}{\sqrt{a}} \psi\left(\frac{t-b}{a}\right) \\
\psi(t)=\text { motherWavelet }
\end{gathered}
$$

The CWT parameters such as ' $a$ ' and ' $\mathrm{b}$ ' (scaling or reciprocal of frequency and translation or time localization or shifting)are continuous. [82], [81]. The main drawback of the CWT is these parameters change continuously and leads to redundancy and impracticality. Due to this, the wavelet will waste its energy to generate a lot of new information. To reduce wasting of effort in the wavelet, the DWT method is considered.

\subsubsection{Discrete Wavelet Transform (DWT)}

According to Daubechies [83] there are two types of DWT, one is based on frames (redundant discrete systems), and another one is based on wavelets. In the case of frames, parameters (dilation and translation) are discrete and chooses any positive or negative integer of powers of one. Due to its redundant description of the original function doesn't support the multi-resolution system, so it is not useful for analyzing physiological signals such as EEG. The other DWT system, called the wavelet-based system, supports multi-resolution analysis. Here, it uses dyadic scales and Positions as replacements of scaling and shift parameters of CWT (shown in Table 4). These are selected based on powers of two. As compared to the CWT, its analysis is efficient[84]. 


$$
D W T(j, k)=) \frac{1}{\sqrt{\left|2^{j}\right|}} \int_{-i n f}^{i n f} x(t) \psi\left(\frac{t-2^{j} k}{2^{j}}\right) d t
$$

TABLE 4: Scaling and Shifting parameters of CWT and DWT.

\begin{tabular}{ccc} 
& CWT & DWT \\
\hline Scaling & $\mathrm{a}$ & $2^{j}$ \\
Shifting & $\mathrm{b}$ & $k 2^{j}$ \\
\hline
\end{tabular}

DWT decomposes only discrete signals for gathering different frequency bands, and this can be done with the help of low and high pass filters of the time-domain signal. Here it follows multi-resolution decomposition for getting the required frequency band signal of the time domain. A signal can be decomposed into many sub-bands, where each sub-band contains a part of the whole signal. The output of the high pass filter is called a complex coefficient, and the output of the low-pass filter is the approximation coefficient [85]. The operational flow of DWT is shown in Fig. 8 to generating the sub-bands. In this process, we must specify the decomposing levels and wavelet function.

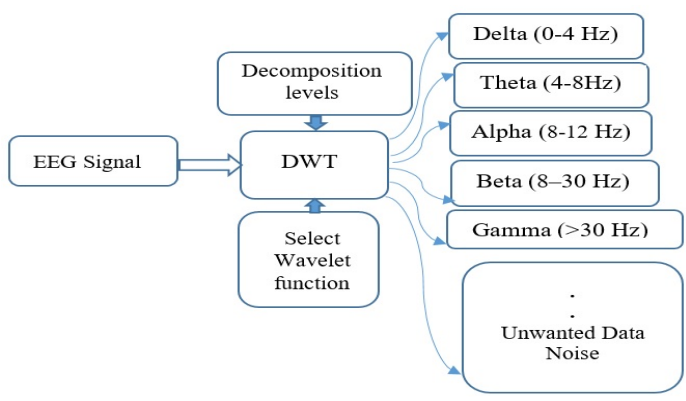

Fig. 8: DWT working flow diagram.

In [85], [86] chooses the decomposition levels based on dominant frequency means; if EEG signals useful frequency bands are below the $30 \mathrm{~Hz}$, then the decomposition levels were 5 . This paper chooses the decomposition level depending on the sampling frequency used to gather the EEG signal. For example, if the sampling frequency is $256 \mathrm{~Hz}$, then the decomposition levels are six. At Decomposition level CA6 (approximate coefficient), we get delta, and at CD6, CD5, CD4, CD3 (Detailed coefficients) levels, we get Theta, Alpha, Beta, and Gamma. The remaining decomposition considers as noise that is at CD2 and CD1.

The decomposition of raw EEG signal for generating sub-bands is shown in Fig. 9. Each step contains two digital filters $g[n]$ is the high pass filter, and $h[n]$ is the low pass filter. The outputs CD, CA are the detailed and approximation coefficients of the EEG signal. The relation between the WT and $H$ and $G$ is

$$
\begin{gathered}
H(z) H\left(z^{-1}\right)+H(-z) H\left(-z^{-1}\right)=1 \\
G(z)=z H\left(-z^{-1}\right)
\end{gathered}
$$

Here $\mathrm{z}$ is H's Z-transform. And G is complementary of $z$-transform displayed in the above equation. In this process,

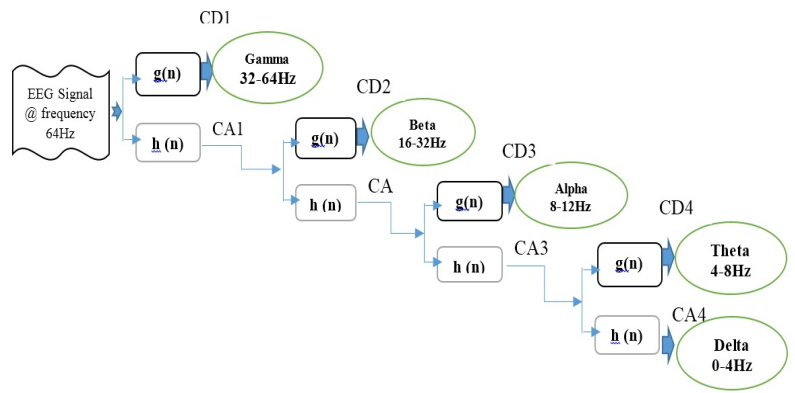

Fig. 9: Sample DWT Decomposition model for EEG signal of $64 \mathrm{~Hz}$ sample frequency.

we are using Multi-Resolution (MR) algorithm because each stage of decomposition uses high pass filter $\mathrm{H}[$.$] and low-$ pass filter G[.]. Again we apply these two filters to the output of the high pass filter. This process is done until we get the required frequency ranges. DWT on EEG signal provides multi-resolution analysis for multiple non-statistical parameters through time, frequency and time-frequency. By this, the subsets of the decomposition coefficients (both approximate and detailed) were used to analyze and predict the EEG subject's behavior. Choosing the best mother wavelet function is another important issue in DWT for generating delta, theta, alpha, beta, and gamma sub-bands. There are multiple wavelet families such as Daubechies, Biorthogonal Coif-let, etc., and each wavelet family contains multiple wavelet functions. The choice of the wavelet transform function depends upon the application, and it must be closely matched to the signal [87].

There are many wavelet families offered in wavelet Transformation showed in Table 5. For knowing the number of wavelet families available in wavelet Transformation in MATLAB, there are some commands such as "wavemnger('read')" or "wavelet families('f')."

TABLE 5: Wavelet Transformation and support Wavelets.

\begin{tabular}{cc} 
Wavelet Family & function \\
\hline Haar & haar \\
Daubechies & db \\
Symlets & sym \\
Coiflets & coif \\
BiorSplines & bior \\
ReverseBior & rbio \\
Meyer & meyr \\
DMeyer & dmey \\
Gaussian & gaus \\
Mexican-hat & mexh \\
Morlet & morl \\
Complex Gaussian & cgau \\
Shannon & shan \\
Frequency B-Spline & fbsp \\
Complex Morlet & cmor \\
Fejer-Korovkin & fk \\
\hline
\end{tabular}

There are many wavelets families, and each wavelet family has different wavelet functions. There are some commands for MATLAB editor for finding the number of wavelet functions in each wavelet family. Those are "wavemngr('read',1)" or "wavelet families('a')" or "wavelet 
families('n')". All wavelet families available in wavelet transformation are not supported by the DWT. A particular wavelet family that supports a DWT operation or not can be determined with the help of one MATLAB command such as "waveinfo('Wavelet function name')," example, "waveinfo('db')." In Table 6 we visualized all wavelet family details that support the DWT procedure.

The decision of wavelet function fundamentally influences the result and selection depending on how smooth the signal and computation involved [88]. However, there are no known methods for choosing a practical wavelet function other than experience means; in many cases, selecting the appropriate Wavelet function is based on experimental result only, means trial and error method. Epileptic seizure detection by analyzing the EEG by considering the multidomain features uses the "db4" Daubechies family wavelet function [89]. They didn't specify the reason for choosing this wavelet function.

In [90], [91] and [88] they uses the wavelet transformation for epileptic seizure detection, but they didn't specify the which wavelet function they used for decomposing the EEG signal to extract the features. For the same application, select "db4" for the mother wavelet function, which was selected based on classification accuracy comparing with few other mother wavelet functions such as Morlet, bior, orthogonal cubic spline, Mexican hat, complex Gaussian, etc. [92]. But all these mother wavelet family functions are not supported by the DWT.

In [93] provide a comparative analysis of wavelet families for EEG. Here they use coiflets (coif1) for analyzing EEG signal, which was selected by compared four mother wavelet functions such as Haar(db1), Daubechies (db2), coiflets (coif1), and Bi-orthogonal (Bior1.1). In this work, they find the best wavelet function by using statistical analysis of classification done through Probabilistic Neural Network (PNN) classifier were equated with Support Vector Machine (SVM) with the assistance of three features such entropy, standard deviation, and energy of different subbands was subtracted using all wavelet functions.

In all the above cases, classification was done for epileptic, so it may be limited to analyzing epileptic EEG signals. It is also a time-consuming process for checking accuracy for every mother wavelet function. In [94] and [95] select the wavelet function using cross-correlation. Hear they specified that "sym9" is best among the forty-five mother wavelet functions of three wavelet families: Daubechies, coiflets, and Symlets. The Same author uses the "sym9" wavelet function in [96] for removing artifacts from EEG signal with the help of Independent Component Analysis with wavelet transform (ICA-WT). Here they didn't check all available mother wavelet functions and computing cross-correlation for the multidimensional signal is the time taken to process, and its value depends on the overall signal, not on the individual samples.

The Wavelet Transmission supports many mother wavelets functions. All these wavelet functions have different filter lengths; long filter length wavelet function required a higher computational cost in case of both time and space compared to the small width filter. Finding the best mother wavelet function through this filter length is not useful for analyzing biomedical signals such as EEG and ECG because analysis of such sensitive signals must be useful to predicting human behavior and finding the diseases even analysis taken more time.

The help of appropriate decomposition levels based on sample frequency and reconstruction of the signal after decomposition finds the best mother wavelet function among all available wavelets till now in the Matlab-2017. For decomposing the signal into a small set of signals, there are many wavelet families explained in Table 6. All these wavelet functions are support by DWT to analyze the biomedical signal such as EEG. To determine the best wavelet function for generating EEG sub-bands, first find the number of levels (L) based on the sampling frequency $\left(F_{s}\right)$ of EEG signal with the help of the following equation.

$$
L=\left\lceil\log _{2}\left(\frac{F_{s}}{4}\right)\right\rceil
$$

Decompose the EEG signals up to L levels using every available mother wavelet function as shown in Fig. 8. After getting sub-brands such as Delta, Theta, Alpha, Beta, and gamma along with unwanted data (greater than $50 \mathrm{~Hz}$ ), reconstruct the signal and compare the reconstructed signal with the original EEG signal, which has less difference that is the best mother wavelet function for generating EEG sub-bands. To find the difference between the reconstructed signal and the original signal, we have many quantitative measurement techniques in signal processing; among them, we choose any method that performs well in less time in the case of both single-dimensional and multidimensional signals. We are choosing Mean Squared Error (MSE). MSE is a quantitative measurement technique used to calculate the degree of similarity between the two signals [97].

MSE of single dimensional signal done with help of the following equation

$$
M S E=\frac{1}{N} \sum_{i=1}^{N}\left(X_{i}-Y_{i}\right)^{2}
$$

MSE of multidimensional signal done with help of the following equation

$$
M S E=\frac{\operatorname{sum}(D(:))}{\text { TotalSamples }}
$$

here

$$
D=a b s(X-Y)^{2}
$$

In this work, we compare the total of one hundred thirtyone (131) wavelet functions of eight wavelet families, which DWT supports (shown in Table 6). Here we use two types of datasets. Standard datasets gathered online from Physionet [98] and BCI competition [99]. Another type of data set is gathered through our device. Here we considered both single-dimensional and multidimensional data sets in two formats such as "edf" and "mat" formats. In edf format the multidimensional datasets are available at [100]. In this work, we used twenty-one datasets; among them, eleven are ".edf" format which was gathered through the online and local hospitals. The remaining ten are ".mat" format; among them, five are gathered online, such as BCI computation websites, and five are gathered directly from the human brain with the help of the available device in our institute. 
TABLE 6: DWT supported Wavelets

\begin{tabular}{|c|c|c|c|l|c|}
\hline Wavelet-family & Short Name & Orthogonal & Biorthogonal & Order N & Example \\
\hline \hline Fejer-Korovkin & $\mathrm{fk}$ & YES & NO & $4,6,8,14,18,22$ & fk4, fk6 \\
Daubechies & $\mathrm{db}$ & YES & YES & 1 to 45 & db1 or haar, db2, db25 \\
Symlets & sym & YES & YES & 1 to 45 & sym1, sym2,sym6, sym8 \\
Coiflets & coif & YES & YES & 1 to 5 & coif2, coif4 \\
Biorthogonal & bior & NO & YES & $1.1,1.3,1.5,2.2,2.4,2.6,2.8,3.1$, & bior 1.1, bior 2.2 \\
& & & & $3.3,3.5,3.7,3.9,4.4,5.5,6.8$ & rbio 1.1, rbio 2.2 \\
Reverse Biorthogonal & rbio & NO & YES & $1.1,1.3,1.5,2.2,2.4,2.6,2.8,3.1$, & r. \\
DMeyer & & & & $3.3,3.5,3.7,3.9,4.4,5.5,6.8$ & dmey \\
\hline
\end{tabular}

In this experiment, we use each wavelet function to decompose the twenty-one datasets and reconstruct the new signal with the help of the decomposed components. After getting the reconstructed signal, we calculate the individual MSE of all datasets. After calculating MSE for every dataset with the same wavelet function, we calculate the mean value of all these MSE's. This is the final MSE value of that particular wavelet function. Initially, we compare all the average MSE values of individual wavelet families. Daubechies Wavelets: Is belongs to the orthogonal wavelets family used in many problems solving techniques related to the signal analysis and it has infinitely supported function [80] and supports vanishing wavelet moments and computed with finite impulse response conjugate mirror filter [101].

These Wavelets are written as "dbN" where $\mathrm{N}$ is from 1 to $45 . d b 1$ is also called haar, and it is related to the Haar transform mathematical operation. In $d b$ family haar is only symmetric wavelet, and its advantages are fast and memory-efficient, and simple[102]. The epilepsy EEG analysis was done with the help of extracting EEG subbands by using Daubechies (db4) wavelet function [103]. We find all forty-five wavelet functions MES's of Daubechies wavelet family. Among them, we plot the graph for the first eleven wavelet functions in ascending order, as shown in Fig. 10.

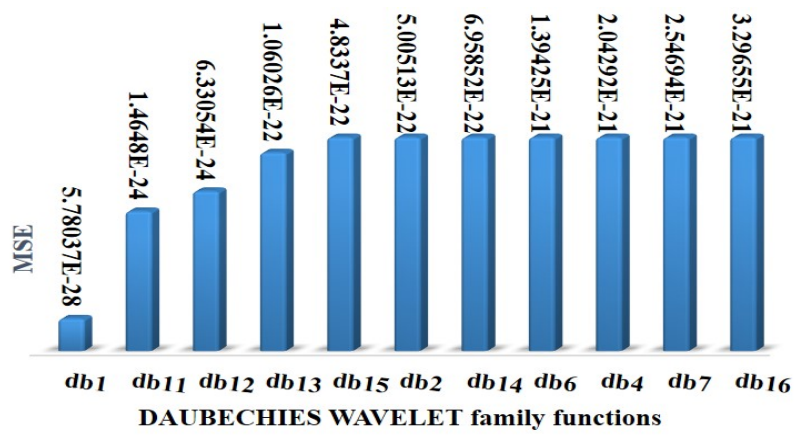

Fig. 10: Evaluation of DAUBECHIES Wavelet functions.

\subsubsection{Coiflets Wavelets}

This is constructed by Daubechies and R.Coifman. It has two functions such as wavelet function has $2 \mathrm{~N}$ movements and scaling function has $2 \mathrm{~N}-1$ to support the length $6 \mathrm{~N}-1$ [104]. Some researchers using coif 1 mother wavelet function analyze the EEG and detect the seizure [105]. Determination of best Coiflets wavelet function is shown in Fig. 11.

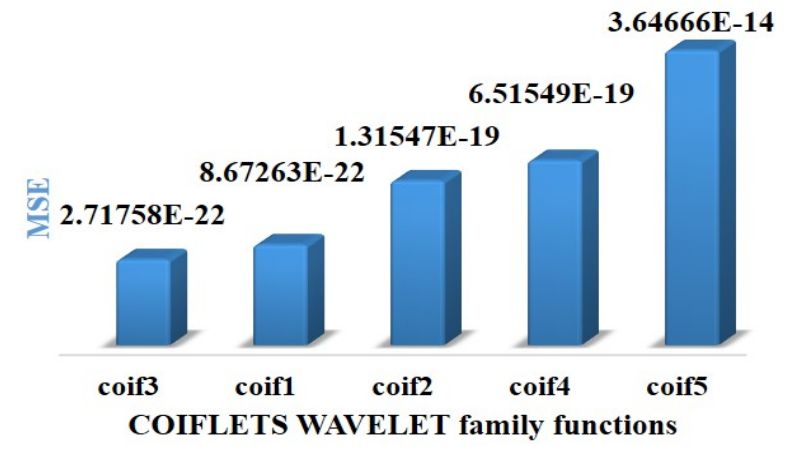

Fig. 11: Evaluation of Coiflets Wavelet functions.

\subsubsection{Symlet Wavelets}

It is similar to the Daubechies family wavelets. "db" and "sym" properties are similar. As like "dbN" symlets also have "symN" Hear $\mathrm{N}$ is from 1 to 45 . These wavelets and their MSE values are shown in Fig. 12. Many researchers use these mother wavelet function for extracting the subbands from EEG signal. Some researchers specifies sym 9 is best among sym 1 to sym 20 [95], some other researcher specifies that sym 7 is best as compared to the sym of order $5,7,8$, and 9 for extracting the features from EEG to identify the dyslexia [106]. In our observation up to "sym35" DWT perform decomposition in less time. After on wards, means from sym36-sym45 Matlab takes more time. First, eleven best sym wavelet functions for analyzing EEG signal is showed in Fig.12.

\subsubsection{Bi-orthogonal Wavelets}

It has the capability to reconstruct and decompose both signal and image. These wavelets support symmetric wavelets. Orthogonal property of wavelet has flexibility for development of wavelet bases. In case of Bi-orthogonal, there is more freedom as compared to orthogonal wavelets by providing two scaling functions useful for multiple multiresolution analysis of two wavelet functions. This feature makes decomposition and reconstruction with help of two different wavelet functions in bi-orthogonal mother wavelet [107]. To decompose EEG signal for extracting the subbands use biorthogonal mother wavelet function for classifying the normal and epileptic human [108], but they didn't specify which wavelet function was used in biorthogonal wavelet family. With the help of bior 4.4 analyze the EEG signal for predicting the epileptic seizure [109], here also 


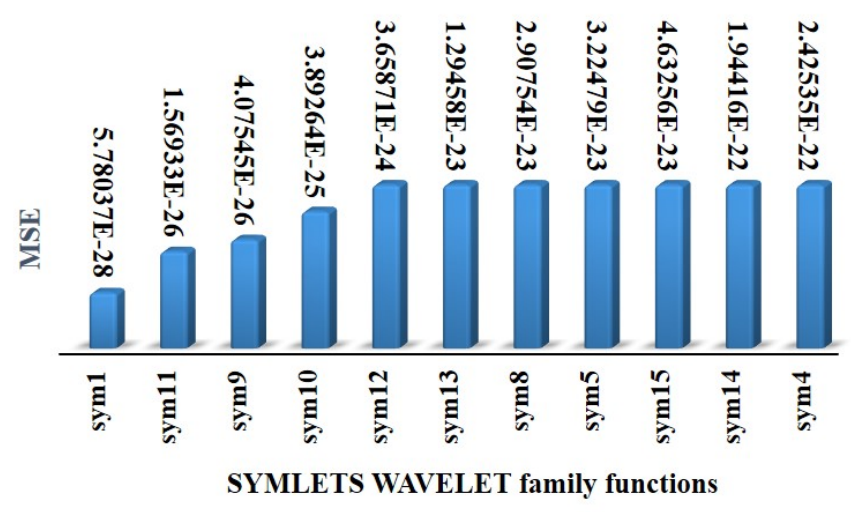

Fig. 12: Evaluation of Symlets Wavelet functions.

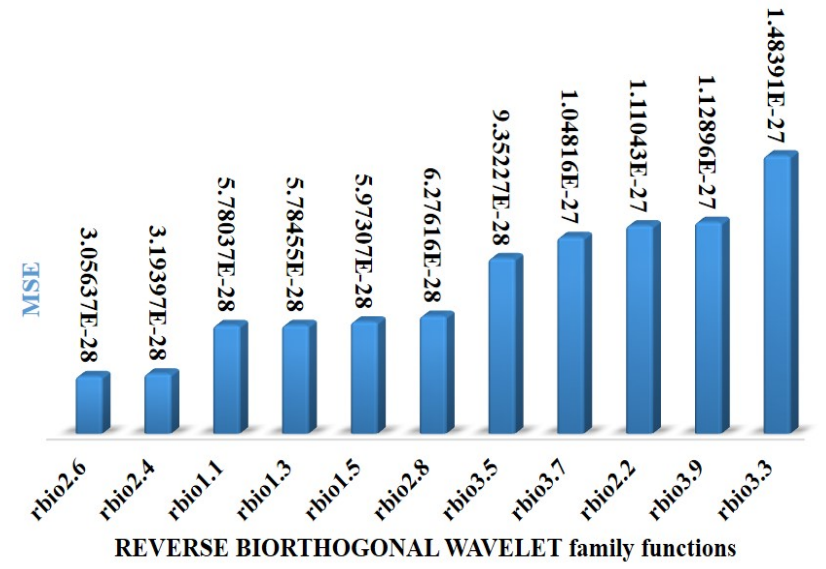

Fig. 14: Evaluation of Reverse-Bi-orthogonal Wavelet functions.

among remaining. In Fig. 15 we compare the all its family wavelet function.

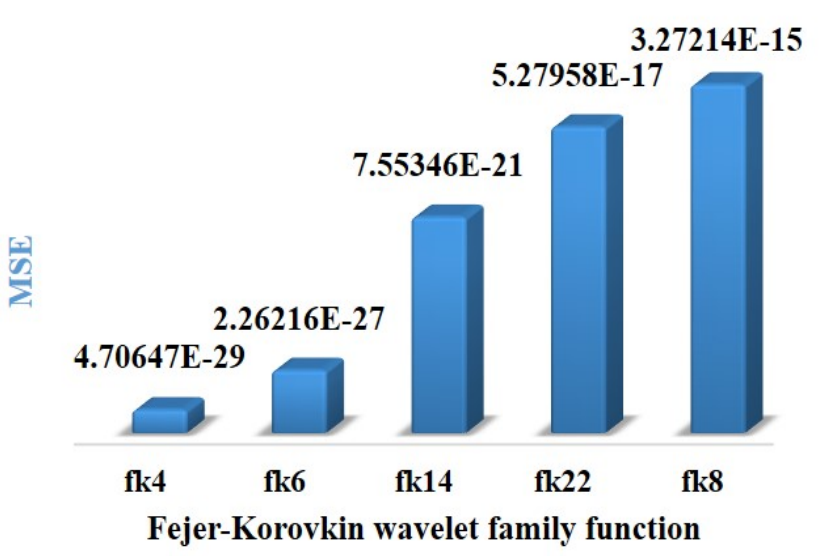

Fig. 15: Evaluation of Fejer-Korovkin Wavelet functions.

\subsubsection{DMeyer Wavelet}

In this wavelet family we have only one wavelet function. It supports both orthogonal and bi-orthogonal operations. Main advantages of this mother wavelet function are symmetry and speed of computation. But it is not suitable for time domain signal [110].In our experiment, it performs very poor for decomposing the signal. In Fig.16 average MSE's of "dmey" wavelet function is displayed.

\section{ILLUSTRATE THE OVERVIEW OF THE RESULT}

For generating these sub-bands, we use some techniques such as Fourier Transform (FFT \& STFT), Digital filters (FIR \& IIR) Wavelet Transform (CWT \& DWT). In the case of Fourier Transformation, problems of FFT are suffer from huge noise sensitivity, and if a signal is linear means stationary, then it performs the operation well, but in the case of the non-stationary signal, its performance is low as compared

\subsubsection{Fejer Korovkin Wavelets}

It is also an orthogonal wavelet family. This family contains only six wavelet functions. Till now no one can use this wavelet function for decomposing the EEG signal. This wavelet function available in latest MATLAB versions only such as 2016a on wards. In our experiment, it performs best 


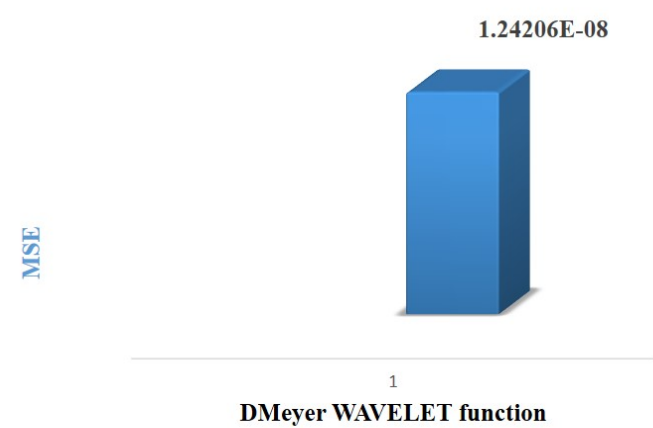

Fig. 16: Evaluation of DMeyer (dmey) Wavelet function.

to the other digital signal processing technique, because some small changes didn't recognize [111]. This means it is the incapability to associate features in the frequency domain with their location in time. Due to this reason in many recent EEG-based applications, such as finding the drowsiness of a driver and estimating the sleepy disorders not preferring FFT. STFT is an extension of FFT for analyzing non-stationary signals with the help of a special window. STFT limitation is if expanding the determination in time reduces the determination in frequency, and vice versa [88]. The window size is fixed; you choose a particular size for the time window, then it will be the same for all the frequencies [112]. If we use a wide window, then it consumes more time. If you use a narrow window, then poor frequency resolution. Coming to Digital filters, the Finite impulse response (FIR) filter is the simplest way to extract the subbands. Among two methods of FIR filters, such as frequency method and windowing method, windowing methods are best for EEG analysis, but the difficulty of this method is, some windows used in this filter are applicable only if the EEG signal is sampled at $1000 \mathrm{~Hz}$. So if the signal is sampled at less frequency like $250 \mathrm{~Hz}$, this method is not suitable. It requires lots of inputs and has a large delay. Another digital filter, Infinite Impulse Response (IIR) overcomes few drawbacks of the FIR filter, like delay. It uses a recursive method for computing required calculations. IIR also has some limitations; if coefficients are not chosen properly, then the IIR filter becomes unstable. In some cases, it is not symmetric. Due to this, different frequencies will appear at the output with a different shift in phases. It is best for getting a single band instead of multiple bands. Because it considers multiple factors and time was taken.

Instead of creating filters (Example: low-pass, band-pass filters) using FIR \& IIR for extracting required rhythms from EEG signal, using these digital filters concepts in Wavelet Transmission to provide multi-resolution methodology gives more accurate results. Wavelet Transformation is best for analyzing the EEG signal because it overcomes all drawbacks of the existing Digital signal processing. Areas of Windows or wavelets which are used by STFT and WT are determined by Heisenberg's inequality. Comparison between FT, STFT, and WT for transforming data is shown in Fig. 18. It is a Time-frequency analysis method, and it
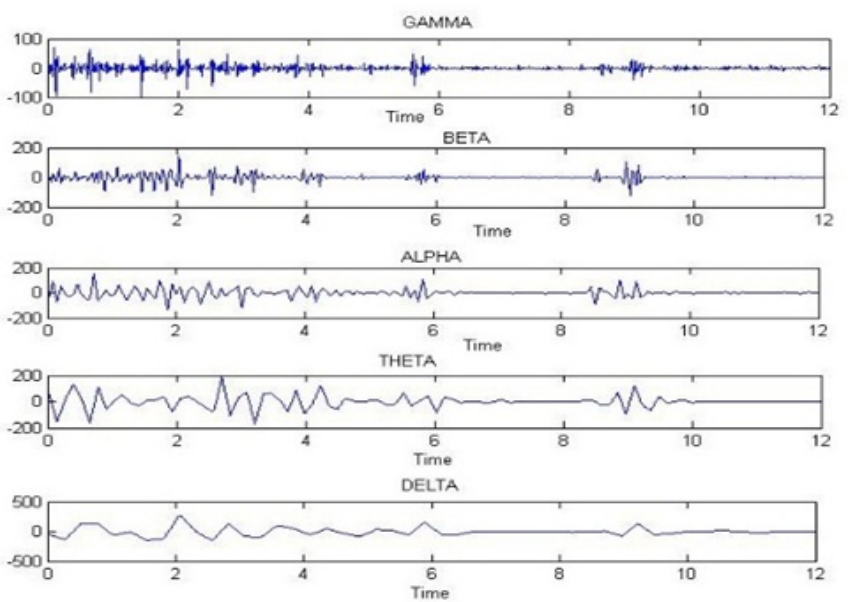

Fig. 17: EEG signal sub-bands generated by DWT.

uses the STFT and Digital filter concept for analyzing the EEG signal.

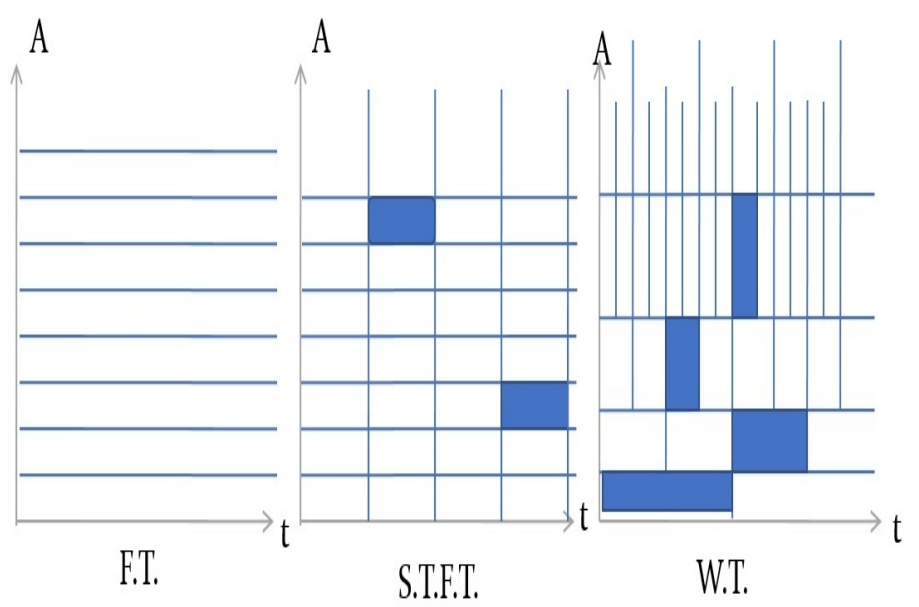

Fig. 18: Comparison between F.T., S.T.F.T., and W.T.

Among two WT methods, DWT is better for extracting the sub-bands from EEG if you know the best wavelet function and decomposition levels. Decomposition levels and finding the best DWT wavelets functions for EEG are discussed in the previous section. Comparing those all wavelets which are supported by the DWT is shown in Fig. 19, it is seen that "fk4" has the least means square error and "bior2.4.1" has second least means square error. From our analysis, "fk4" and "bior2.4" are best for generating sub-bands. Here "fk4" supports only orthogonal, but bi-orthogonal supports both orthogonal and bi-orthogonal operations. So among all the DWT wavelet functions, Biorthogonal is best because, in Bi-orthogonal, there are separate wavelets for decomposition and reconstruction. Biorthogonal $M S E$ value is also very small, so it is preferable for EEG analysis. Table 7 provides the summary of the different methodologies used for sub-band generations in terms of their strengths and weaknesses. The EEG subbands are generated by using the DWT represented in Fig. 19. 


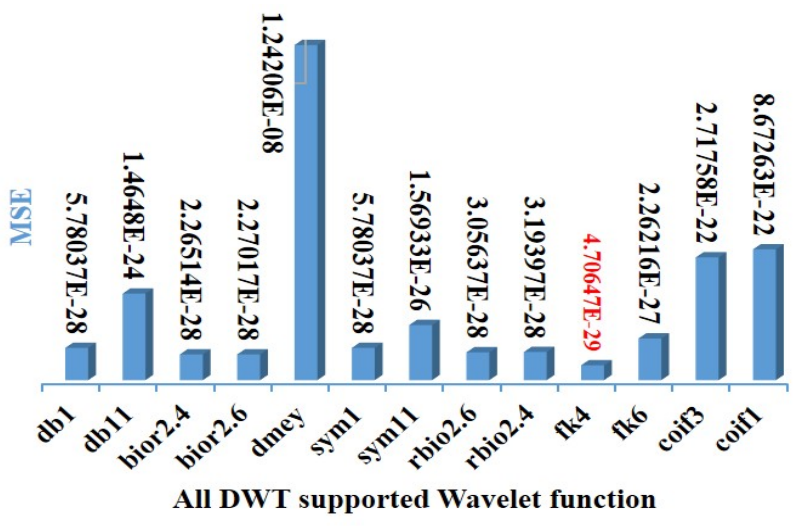

Fig. 19: Evaluation of overall DWT Wavelet functions.

TABLE 7: Different EEG signal analysis methods

\begin{tabular}{|c|c|c|c|c|}
\hline \multicolumn{3}{|c|}{ Method } & Strengths & Weakness \\
\hline \multirow[t]{2}{*}{ FT } & & FFT & Easy to use. Fast computing & $\begin{array}{l}\text { Suffer from huge noise ser } \\
\text { only for stationary signals }\end{array}$ \\
\hline & & STFT & Supports Non-stationary signals & $\begin{array}{l}\text { Fixed size window, if you chosese wide win- } \\
\text { dow, consumes more time and if you choose } \\
\text { narrow window leads poor frequency reso- } \\
\text { lution }\end{array}$ \\
\hline \multirow{3}{*}{ Digital Filters } & FIR & $\begin{array}{l}\text { Fre- } \\
\text { quency }\end{array}$ & $\begin{array}{l}\text { Simple when desired frequency response has } \\
\text { been specified, Easy to use as like FT Fand } \\
\text { supports any given magnitude response. }\end{array}$ & $\begin{array}{l}\text { Length of filter is long and results are not } \\
\text { optimal }\end{array}$ \\
\hline & & Window & $\begin{array}{l}\text { Relatively simple as compared to the other } \\
\text { method ease of use, little design flexibility }\end{array}$ & $\begin{array}{l}\text { Application only if frequency response } \\
\text { specification is absolutely integral. }\end{array}$ \\
\hline & & $\Pi \mathrm{IR}$ & more efficient and supports recursive operations & limited \\
\hline \multirow[t]{2}{*}{ WT } & & CWT & $\begin{array}{l}\text { Works at any scale and any translation point, } \\
\text { simple in case of detecting small features }\end{array}$ & $\begin{array}{l}\text { analog ssystem } \\
\text { lighiteduces too much of data and } \\
\text { limited to continuous signal }\end{array}$ \\
\hline & & DWT & $\begin{array}{l}\text { Supports both orthogonal and biorthogonal. } \\
\text { Computational time is very less }\end{array}$ & $\begin{array}{l}\text { Choosing decomposition levels and selec- } \\
\text { tion of mother wavelet function are not } \\
\text { clear. }\end{array}$ \\
\hline
\end{tabular}

\section{Conclusion \& future Scope}

Analyzing EEG signals is difficult due to its non-stationary nature for acquiring the required information to predict human behavior in response to a certain event. Although increasingly employed in various signal processing techniques for extracting required sub-bands from EEG signals, there is no certain explanation which one of these techniques is better in terms of cost (in terms of time and space) and accuracy (in terms of performance). This paper covers such topics and different topographic systems (10-20 systems) for extracting EEG from the human brain. Also, we studied EEG challenges and their role in BCI, use of sub-band generation, and applications. Various state-of-art techniques are compared related to FFT, STFT, FIR, IIR, CWT, and DWT for extracting the EEG sub-bands. Every method has its strengths and limitations, which we choose based on different situations and applications. This study showed several limitations of these methods, and we concentrate more on the DWT method of WT weaknesses like choosing better Mother Wavelet Function $(M W T)$ and decomposition levels. Here we choose de-composition levels based on sample frequency with the help of EEG sub-band range and select the mother wavelet function among all 131 mother wavelets functions supported by DWT using mean square error analysis. From the result, "fk4" and "bior2.4" showed better performance, but fk4 supports only orthogonal computation, and bior2.4 supports both orthogonal and bi-orthogonal computation. In future work, we would like to study other wavelet transforms related to DWT, such as MODWT, Multi-signal 1-D wavelet decomposition $(M D W T D E C)$, and SWT use them in case of sub-band generation in EEG.

\section{REFERENCES}

[1] J. A. Chambers Saeid Sanei. EEG signal processing. John Wiley and Sons, 2007.

[2] Mohammad-Parsa Hosseini, Amin Hosseini, and Kiarash Ahi. A review on machine learning for eeg signal processing in bioengineering. IEEE reviews in biomedical engineering, 14:204-218, 2020.

[3] Michal Teplan et al. Fundamentals of eeg measurement. Measurement science review, 2(2):1-11, 2002.

[4] Khansa Rasheed, Adnan Qayyum, Junaid Qadir, Shobi Sivathamboo, Patrick Kwan, Levin Kuhlmann, Terence OBrien, and Adeel Razi. Machine learning for predicting epileptic seizures using eeg signals: A review. IEEE Reviews in Biomedical Engineering, 14:139-155, 2020

[5] Mark H Libenson. Practical approach to electroencephalography. Elsevier Health Sciences, 2012.

[6] Ira J Rampil. A primer for eeg signal processing in anesthesia. Anesthesiology: The Journal of the American Society of Anesthesiologists, 89(4):980-1002, 1998.

[7] Lun-De Liao, Chin-Teng Lin, Kaleb McDowell, Alma E Wickenden, Klaus Gramann, Tzyy-Ping Jung, Li-Wei Ko, and JyhYeong Chang. Biosensor technologies for augmented braincomputer interfaces in the next decades. Proceedings of the IEEE, 100(Special Centennial Issue):1553-1566, 2012.

[8] Supriya Supriya, Siuly Siuly, Hua Wang, and Yanchun Zhang. Epilepsy detection from eeg using complex network techniques: A review. IEEE Reviews in Biomedical Engineering, 2021.

[9] Seungchan Lee, Younghak Shin, Soogil Woo, Kiseon Kim, and Heung-No Lee. Review of wireless brain-computer interface systems. In Brain-Computer Interface Systems-Recent Progress and Future Prospects. InTech, 2013.

[10] Lawrence Ashley Farwell and Emanuel Donchin. Talking off the top of your head: toward a mental prosthesis utilizing eventrelated brain potentials. Electroencephalography and clinical Neurophysiology, 70(6):510-523, 1988.

[11] Kamel Nidal and Aamir Saeed Malik. EEG/ERP Analysis: Methods and Applications. CRC Press, 2014.

[12] Zohreh Zakeri, Mohammad Reza Haji Samadi, Neil Cooke, and Peter Jancovic. Automatic erp classification in eeg recordings from task-related independent components. In Biomedical and Health Informatics (BHI), 2016 IEEE-EMBS International Conference on, pages 288-291. IEEE, 2016.

[13] Salman Mohd Khan, Abid Ali Khan, and Omar Farooq. Selection of features and classifiers for emg-eeg-based upper limb assistive devicesa review. IEEE reviews in biomedical engineering, 13:248-260, 2019

[14] Valentina Bono, Dwaipayan Biswas, Saptarshi Das, and Koushik Maharatna. Classifying human emotional states using wireless eeg based erp and functional connectivity measures. In Biomedical and Health Informatics (BHI), 2016 IEEE-EMBS International Conference on, pages 200-203. IEEE, 2016.

[15] Benjamin Blankertz, Florin Popescu, Matthias Krauledat, Siamac Fazli, Michael Tangermann, K Müller, R Grinter, T Rodden, $\mathrm{P}$ Aoki, E Cutrell, et al. Challenges for brain-computer interface research for human-computer interaction applications. In ACM CHI Workshop on Brain-Computer Interfaces for HCI and Games, 2008.

[16] Qin Shuren and Ji Zhong. Extraction of features in eeg signals with the non-stationary signal analysis technology. In Engineering in Medicine and Biology Society, 2004. IEMBS'04. 26th Annual International Conference of the IEEE, volume 1, pages 349-352. IEEE, 2004.

[17] Alexander Ya Kaplan, Andrew A Fingelkurts, Alexander A Fingelkurts, Sergei V Borisov, and Boris S Darkhovsky. Nonstationary nature of the brain activity as revealed by eeg/meg: methodological, practical and conceptual challenges. Signal processing, 85(11):2190-2212, 2005.

[18] John S Barlow. Methods of analysis of nonstationary eegs, with emphasis on segmentation techniques: a comparative review. Journal of Clinical Neurophysiology, 2(3):267-304, 1985.

[19] Jonathan R Wolpaw, Niels Birbaumer, Dennis J McFarland, Gert Pfurtscheller, and Theresa M Vaughan. Brain-computer interfaces for communication and control. Clinical neurophysiology, 113(6):767-791, 2002

[20] Gerwin Schalk and Eric C Leuthardt. Brain-computer interfaces using electrocorticographic signals. IEEE reviews in biomedical engineering, 4:140-154, 2011. 
[21] Ashwin K Whitchurch, Jose K Abraham, Meghana A Lonkar, and Vijay K Varadan. Design of a compact amplifier and signal conditioning module for wireless eeg monitoring. In Region 5 Technical Conference, 2007 IEEE, pages 153-156. IEEE, 2007.

[22] Eleanor A Curran and Maria J Stokes. Learning to control brain activity: a review of the production and control of eeg components for driving brain-computer interface (bci) systems. Brain and cognition, 51(3):326-336, 2003.

[23] Steven G Mason and Gary E Birch. A general framework for brain-computer interface design. IEEE transactions on neural systems and rehabilitation engineering, 11(1):70-85, 2003.

[24] Ali Bashashati, Mehrdad Fatourechi, Rabab K Ward, and Gary E Birch. A survey of signal processing algorithms in braincomputer interfaces based on electrical brain signals. Journal of Neural engineering, 4(2):R32, 2007.

[25] Jacques J Vidal. Real-time detection of brain events in eeg. Proceedings of the IEEE, 65(5):633-641, 1977.

[26] William D Penny and Karl J Friston. Functional imaging. Scholarpedia, 2(5):1478, 2007.

[27] Bruce Crosson, Anastasia Ford, Keith M McGregor, Marcus Meinzer, Sergey Cheshkov, Xiufeng Li, Delaina Walker-Batson, and Richard W Briggs. Functional imaging and related techniques: An introduction for rehabilitation researchers. Journal of rehabilitation research and development, 47(2):vii, 2010.

[28] Thomas N Lal, Thilo Hinterberger, Guido Widman, Michael Schröder, N Jeremy Hill, Wolfgang Rosenstiel, Christian E Elger, Niels Birbaumer, and Bernhard Schölkopf. Methods towards invasive human brain computer interfaces. In Advances in neural information processing systems, pages 737-744, 2005.

[29] Febo Cincotti, Donatella Mattia, Fabio Aloise, Simona Bufalari, Gerwin Schalk, Giuseppe Oriolo, Andrea Cherubini, Maria Grazia Marciani, and Fabio Babiloni. Non-invasive braincomputer interface system: towards its application as assistive technology. Brain research bulletin, 75(6):796-803, 2008.

[30] Xiaogang Chen, Yijun Wang, Masaki Nakanishi, Xiaorong Gao, Tzyy-Ping Jung, and Shangkai Gao. High-speed spelling with a noninvasive brain-computer interface. Proceedings of the national academy of sciences, 112(44):E6058-E6067, 2015.

[31] Md Kafiul Islam, Amir Rastegarnia, and Zhi Yang. Methods for artifact detection and removal from scalp eeg: A review. Neurophysiologie Clinique/Clinical Neurophysiology, 46(4):287-305, 2016.

[32] Rodney J Croft and Robert J Barry. Removal of ocular artifact from the eeg: a review. Neurophysiologie Clinique/Clinical Neurophysiology, 30(1):5-19, 2000.

[33] Wen Qi. Eog artifacts removal in eeg measurements for affective interaction with brain computer interface. In Intelligent Information Hiding and Multimedia Signal Processing (IIH-MSP), 2012 Eighth International Conference on, pages 471-475. IEEE, 2012.

[34] Qinglin Zhao, Bin Hu, Yujun Shi, Yang Li, Philip Moore, Minghou Sun, and Hong Peng. Automatic identification and removal of ocular artifacts in eeg?improved adaptive predictor filtering for portable applications. IEEE transactions on nanobioscience, 13(2):109-117, 2014.

[35] Alois Schlögl, Claudia Keinrath, Doris Zimmermann, Reinhold Scherer, Robert Leeb, and Gert Pfurtscheller. A fully automated correction method of eog artifacts in eeg recordings. Clinical neurophysiology, 118(1):98-104, 2007.

[36] Hans Hallez, Maarten De Vos, Bart Vanrumste, Peter Van Hese, Sara Assecondi, Koen Van Laere, Patrick Dupont, Wim Van Paesschen, Sabine Van Huffel, and Ignace Lemahieu. Removing muscle and eye artifacts using blind source separation techniques in ictal eeg source imaging. Clinical Neurophysiology, 120(7):1262-1272, 2009.

[37] Valer Jurcak, Daisuke Tsuzuki, and Ippeita Dan. 10/20, 10/10, and 10/5 systems revisited: their validity as relative headsurface-based positioning systems. Neuroimage, 34(4):1600-1611, 2007.

[38] C Vaidyanathan and KM Buckley. A sampling theorem for eeg electrode configuration. IEEE transactions on biomedical engineering, 44(1):94-97, 1997.

[39] George H Klem, Hans Otto Lüders, HH Jasper, C Elger, et al. The ten-twenty electrode system of the international federation. Electroencephalogr Clin Neurophysiol, 52(3):3-6, 1999.

[40] GE Chatrian, E Lettich, and PL Nelson. Ten percent electrode system for topographic studies of spontaneous and evoked eeg activities. American Journal of EEG technology, 25(2):83-92, 1985.
[41] Robert Oostenveld and Peter Praamstra. The five percent electrode system for high-resolution eeg and erp measurements. Clinical neurophysiology, 112(4):713-719, 2001.

[42] Sabbir Ibn Arman, Arif Ahmed, and Anas Syed. Cost-effective eeg signal acquisition and recording system. International Journal of Bioscience, Biochemistry and Bioinformatics, 2(5):301, 2012.

[43] Marc R Nuwer, Giancarlo Comi, Ronald Emerson, A FuglsangFrederiksen, JM Guerit, H Hinrichs, A Ikeda, FJ Luccas, and P Rappelsberger. If $\mathrm{cn}$ standards for digital recording of clinical eeg. the international federation of clinical neurophysiology. Electroencephalography and clinical neurophysiology. Supplement, 52:11, 1999.

[44] Electroencephalography 10/20 System positioning Manual. Trans Cranial Technologies, Available at: https://www.trans-cranial.com/local/manuals/10_20_pos_ man_v1_0_pdf.pdf/, 2012.

[45] Electroencephalography I Laboratory. CleveLabs Laboratory Course System - Teacher Edition, Available at:. https:// glneurotech.com/docrepo/teaching-labs/EEG_I_Teacher.pdf/, 2006.

[46] Jaakko Malmivuo, Sari Ahokas, and Toni Välkky. High-resolution eeg recording system using smart electrodes. In Electronic Conference (BEC), 2014 14th Biennial Baltic, pages 21-24. IEEE, 2014.

[47] Christoph S Herrmann, Maren Grigutsch, and Niko A Busch. 11 eeg oscillations and wavelet analysis. Event-related potentials: A methods handbook, page 229, 2005.

[48] Steven L Fahrion, E Dale Walters, Lolafaye Coyne, and Thomas Allen. Alterations in eeg amplitude, personality factors, and brain electrical mapping after alpha-theta brainwave training: A controlled case study of an alcoholic in recovery. Alcoholism: Clinical and Experimental Research, 16(3):547-552, 1992.

[49] The McGill Physiology Virtual Lab. Biomedical Signals Acquisition. http://www.medicine.mcgill.ca/physio/vlab/biomed_ signals/eeg_n.htm/, 2017.

[50] Ernst Niedermeyer and FH Lopes da Silva. Electroencephalography: basic principles, clinical applications, and related fields. Lippincott Williams \& Wilkins, 2005.

[51] Valérie Billard, Pedro L Gambus, Nassib Chamoun, Donald R Stanski, and Steven L Shafer. A comparison of spectral edge, delta power, and bispectral index as eeg measures of alfentanil, propofol, and midazolam drug effect. Clinical Pharmacology $\mathcal{E}$ Therapeutics, 61(1):45-58, 1997.

[52] Alexander A Borbély, Fritz Baumann, Daniel Brandeis, Inge Strauch, and Dietrich Lehmann. Sleep deprivation: effect on sleep stages and eeg power density in man. Electroencephalography and clinical neurophysiology, 51(5):483-493, 1981.

[53] Daniel L Schacter. Eeg theta waves and psychological phenomena: A review and analysis. Biological psychology, 5(1):47-82, 1977.

[54] Mark J Russ, Scott S Campbell, Tatsuyuki Kakuma, Katherine Harrison, and Elizabeth Zanine. Eeg theta activity and pain insensitivity in self-injurious borderline patients. Psychiatry research, 89(3):201-214, 1999.

[55] Koji NAKASHIMA and Haruhiko SATO. The effects of various mental tasks on appearance of frontal midline theta activity in eeg. Journal of human ergology, 21(2):201-206, 1992.

[56] Kei Omata, Takashi Hanakawa, Masako Morimoto, and Manabu Honda. Spontaneous slow fluctuation of eeg alpha rhythm reflects activity in deep-brain structures: a simultaneous eeg-fmri study. PloS one, 8(6):e66869, 2013.

[57] Andreas Fink and Mathias Benedek. Eeg alpha power and creative ideation. Neuroscience \& Biobehavioral Reviews, 44:111-123, 2014.

[58] Wolfgang Klimesch. Eeg-alpha rhythms and memory processes. International Journal of Psychophysiology, 26(1):319-340, 1997.

[59] Andreas K Engel and Pascal Fries. Beta-band oscillationssignalling the status quo? Current opinion in neurobiology, 20(2):156-165, 2010.

[60] Manuel Zaepffel, Romain Trachel, Bjørg Elisabeth Kilavik, and Thomas Brochier. Modulations of eeg beta power during planning and execution of grasping movements. PloS one, 8(3):e60060, 2013.

[61] Xiaoxuan Jia and Adam Kohn. Gamma rhythms in the brain. PLoS Biol, 9(4):e1001045, 2011.

[62] Binaural Beats Meditation. All the Benefits of Meditation at the Touch of a Button. https://www.binauralbeatsmeditation.com/ benefits-gamma-waves/, 2017. 
[63] E Oran Brigham. The fast fourier transform and its applications. UK: Prentice Hall, 1988.

[64] Mathuranathan Viswanathan. Simulation of digital communication systems using matlab. Mathuranathan Viswanathan at Smashwords, 2013.

[65] Wojciech Sałabun. Processing and spectral analysis of the raw eeg signal from the mindwave. Przeglad Elektrotechniczny, 90(2):169-174, 2014

[66] Pierre Duhamel and Martin Vetterli. Fast fourier transforms: a tutorial review and a state of the art. Signal processing, 19(4):259-299, 1990

[67] M Murugappan, Subbulakshmi Murugappan, Celestin Gerard, et al. Wireless eeg signals based neuromarketing system using fast fourier transform (fft). In Signal Processing $\mathcal{E}$ its Applications (CSPA), 2014 IEEE 10th International Colloquium on, pages 25-30. IEEE, 2014.

[68] Abdul-Bary Raouf Suleiman and Toka Abdul-Hameed Fatehi. Features extraction techniqes of eeg signal for bci applications. Faculty of Computer and Information Engineering Department College of Electronics Engineering, University of Mosul, Iraq, 2007.

[69] Leland B Jackson. Digital Filters and Signal Processing: With MATLAB® Exercises. Springer Science \& Business Media, 2013.

[70] Arojit Roychowdhury. Fir filter design techniques. Electronic Systems Group, EE Dept, IIT Bombay, 2002.

[71] Chen-Long Hu. Design and verification of fir filter based on matlab and dsp. In Image Analysis and Signal Processing (IASP), 2012 International Conference on, pages 1-4. IEEE, 2012.

[72] Nova Eka Diana, Umi Kalsum, Ahmad Sabiq, Wisnu Jatmiko, and Petrus Mursanto. Comparing windowing methods on finite impulse response (fir) filter algorithm in electroencephalography (eeg) data processing. Journal of Theoretical and Applied Information Technology, 88(3):558, 2016.

[73] EA Mahmoud, AH Abdalla, AM Wedaa, and MA Hassan. A comparison between windowing fir filters for extracting the eeg components. J Biosens Bioelectron, 6(191):2, 2015.

[74] Douglas F Elliott. Handbook of digital signal processing: engineering applications. Academic press, 2013.

[75] Andreas Widmann, Erich Schröger, and Burkhard Maess. Digital filter design for electrophysiological data-a practical approach. Journal of neuroscience methods, 250:34-46, 2015.

[76] Metin Akay. Wavelet applications in medicine. IEEE spectrum, 34(5):50-56, 1997.

[77] Michael Unser and Akram Aldroubi. A review of wavelets in biomedical applications. Proceedings of the IEEE, 84(4):626-638, 1996.

[78] Dean Cvetkovic, Elif Derya Übeyli, and Irena Cosic. Wavelet transform feature extraction from human ppg, ecg, and eeg signal responses to elf pemf exposures: A pilot study. Digital signal processing, 18(5):861-874, 2008.

[79] Leif Sörnmo and Pablo Laguna. Bioelectrical signal processing in cardiac and neurological applications, volume 8. Academic Press, 2005.

[80] Daniel TL Lee and Akio Yamamoto. Wavelet analysis: theory and applications. Hewlett Packard journal, 45:44-44, 1994.

[81] Kayvan Najarian and Robert Splinter. Biomedical signal and image processing. CRC press, 2005.

[82] M Kemal Kıymik, Inan Güler, Alper Dizibüyük, and Mehmet Akın. Comparison of stft and wavelet transform methods in determining epileptic seizure activity in eeg signals for real-time application. Computers in biology and medicine, 35(7):603-616, 2005.

[83] Ingrid Daubechies. Ten lectures on wavelets, vol. 61 of cbms-nsf regional conference series in applied mathematics, 1992.

[84] Hasan Ocak. Automatic detection of epileptic seizures in eeg using discrete wavelet transform and approximate entropy. Expert Systems with Applications, 36(2):2027-2036, 2009.

[85] Abdulhamit Subasi. Eeg signal classification using wavelet feature extraction and a mixture of expert model. Expert Systems with Applications, 32(4):1084-1093, 2007.

[86] Abdulhamit Subasi and M Ismail Gursoy. Eeg signal classification using pca, ica, lda and support vector machines. Expert Systems with Applications, 37(12):8659-8666, 2010.

[87] Rajeev Agarwal and Jean Gotman. Adaptive segmentation of electroencephalographic data using a nonlinear energy operator. In Circuits and Systems, 1999. ISCAS'99. Proceedings of the 1999 IEEE International Symposium on, volume 4, pages 199-202. IEEE, 1999.
[88] Lalit M Patnaik and Ohil K Manyam. Epileptic eeg detection using neural networks and post-classification. Computer methods and programs in biomedicine, 91(2):100-109, 2008.

[89] Lina Wang, Weining Xue, Yang Li, Meilin Luo, Jie Huang, Weigang Cui, and Chao Huang. Automatic epileptic seizure detection in eeg signals using multi-domain feature extraction and nonlinear analysis. Entropy, 19(6):222, 2017.

[90] Guangyi Chen. Automatic eeg seizure detection using dual-tree complex wavelet-fourier features. Expert Systems with Applications, 41(5):2391-2394, 2014.

[91] Hasan Ocak. Optimal classification of epileptic seizures in eeg using wavelet analysis and genetic algorithm. Signal processing, 88(7):1858-1867, 2008

[92] Oliver Faust, U Rajendra Acharya, Hojjat Adeli, and Amir Adeli. Wavelet-based eeg processing for computer-aided seizure detection and epilepsy diagnosis. Seizure, 26:56-64, 2015.

[93] Tapan Gandhi, Bijay Ketan Panigrahi, and Sneh Anand. A comparative study of wavelet families for eeg signal classification. Neurocomputing, 74(17):3051-3057, 2011.

[94] Noor Kamal Al-Qazzaz, Sawal Ali, Siti Anom Ahmad, Md Shabiul Islam, and Mohd Izhar Ariff. Selection of mother wavelets thresholding methods in denoising multi-channel eeg signals during working memory task. In Biomedical Engineering and Sciences (IECBES), 2014 IEEE Conference on, pages 214-219. IEEE, 2014.

[95] Noor Kamal Al-Qazzaz, Sawal Hamid Bin Mohd Ali, Siti Anom Ahmad, Mohd Shabiul Islam, and Javier Escudero. Selection of mother wavelet functions for multi-channel eeg signal analysis during a working memory task. Sensors, 15(11):29015-29035, 2015.

[96] Noor Kamal Al-Qazzaz, Sawal Hamid Bin Mohd Ali, Siti Anom Ahmad, Mohd Shabiul Islam, and Javier Escudero. Automatic artifact removal in eeg of normal and demented individuals using ica-wt during working memory tasks. Sensors, 17(6):1326, 2017.

[97] Z Wan and AC Bovik. Mean squared error: Love it or leave it? IEEE Signal Processing Magazine, pages 98-117, 2009.

[98] A. L. Goldberger, L. A. N. Amaral, L. Glass, J. M. Hausdorff, P. Ch. Ivanov, R. G. Mark, J. E. Mietus, G. B. Moody, C.-K. Peng, and H. E. Stanley. PhysioBank, PhysioToolkit, and PhysioNet: Components of a new research resource for complex physiologic signals. Circulation, 101(23):e215-e220, 2000 (June 13). Circulation Electronic Pages: http://circ.ahajournals.org/content/101/23/e215.full PMID:1085218; doi: 10.1161/01.CIR.101.23.e215.

[99] Benjamin Blankertz, K-R Muller, Dean J Krusienski, Gerwin Schalk, Jonathan R Wolpaw, Alois Schlogl, Gert Pfurtscheller, Jd R Millan, Michael Schroder, and Niels Birbaumer. The bci competition iii: Validating alternative approaches to actual bci problems. IEEE transactions on neural systems and rehabilitation engineering, 14(2):153-159, 2006.

[100] Bob Kemp. MCH-Westeinde Hospital, Den Haag, The Netherlands. https://www.physionet.org/physiobank/ database/sleep-edfx/?C=M;O=A/, 2013.

[101] Stephane Mallat. A wavelet tour of signal processing: the sparse way. Academic press, 2008

[102] Mohammed A Salem, Nivin Ghamry, and Beate Meffert. Daubechies versus biorthogonal wavelets for moving object detection in traffic monitoring systems. 2009.

[103] Ibrahim Omerhodzic, Samir Avdakovic, Amir Nuhanovic, and Kemal Dizdarevic. Energy distribution of eeg signals: Eeg signal wavelet-neural network classifier. arXiv preprint arXiv:1307.7897, 2013

[104] Abhinav Dixit and Swatilekha Majumdar. Comparative analysis of coiflet and daubechies wavelets using global threshold for image denoising. International Journal of Advances in Engineering E Technology, 6(5):2247, 2013.

[105] Piyush Swami, Manvir Bhatia, Sneh Anand, Bijaya K Panigrahi, and Jayasree Santhosh. Svm based automated eeg seizure detection using ?coiflets? wavelet packets. In Recent Developments in Control, Automation and Power Engineering (RDCAPE), 2015 International Conference on, pages 238-242. IEEE, 2015.

[106] Z Mahmoodin, NS Jalalludin, W Mansor, Khuan Y Lee, and NB Mohamad. Selection of symlets wavelet function order for eeg signal feature extraction in children with dyslexia. In Biomedical Engineering \& Sciences (ISSBES), 2015 IEEE Student Symposium in, pages 113-117. IEEE, 2015. 
[107] Cristina Stolojescu, Ion Railean, Sorin Moga, and Alexandru Isar. Comparison of wavelet families with application to wimax traffic forecasting. In Optimization of Electrical and Electronic Equipment (OPTIM), 2010 12th International Conference on, pages 932-937. IEEE, 2010.

[108] R Kumari and J Jose. Seizure detection in eeg using biorthogonal wavelet and fuzzy $\mathrm{knn}$ classifier. Elixir Hum. Physiol, 41:5766-5770, 2011.

[109] Dinesh Bhati, Manish Sharma, Ram Bilas Pachori, and Vikram M Gadre. Time-frequency localized three-band biorthogonal wavelet filter bank using semidefinite relaxation and nonlinear least squares with epileptic seizure eeg signal classification. Digital Signal Processing, 62:259-273, 2017.

[110] Jesse Sherwood and Reza Derakhshani. On classifiability of wavelet features for eeg-based brain-computer interfaces. In Neural Networks, 2009. IJCNN 2009. International Joint Conference on, pages 2895-2902. IEEE, 2009.

[111] M Akin. Comparison of wavelet transform and fft methods in the analysis of eeg signals. Journal of medical systems, 26(3):241-247, 2002.

[112] Usman Seljuq, Faraz Himayun, and Haroon Rasheed. Selection of an optimal mother wavelet basis function for ecg signal denoising. In Multi-Topic Conference (INMIC), 2014 IEEE 17th International, pages 26-30. IEEE, 2014. 